\title{
Consumo de energía eléctrica y Fuel Oíl en una empresa de productos cárnicos en Cuba
}

\section{Consumption of electrical energy and Fuel 0íl in a meat products company in Cuba}

\author{
(iD) 'Yoandro Rodríguez-Ponce (iD 'Yans Guardia-Puebla (iD) ${ }^{2}$ Manuel Camps-Michelena \\ iD 'uis Alberto Taramona-Ruiz iD 'Eva Sánchez Garcia \\ Universidad de Granma, Cuba.' \\ Universidad Politécnica de Madrid, España. ${ }^{2}$ \\ Universidad Le Cordon Bleu, Lima-Perú. ${ }^{3}$
}

\section{Resumen}

Este estudio tuvo como objetivo determinar el consumo de energía eléctrica y Fuel Oíl de una empresa de producción de cárnicos en Cuba. Se analizaron informaciones existentes de producción y consumos de portadores energéticos en un periodo consecutivo de 36 meses, aplicando las herramientas de la Tecnología de Gestión Total y Eficiente de la Energía (TGTEE), para establecer el consumo de energía eléctrica y Fuel Oíl y se diseñó un biodigestor de cúpula fija para la producción de biogás. La energía eléctrica y Fuel Oíl fueron los mayores influyentes en el costo energético de la producción, representando un 91.93 \% del total del consumo en el período evaluado. Se obtuvieron $15 \mathrm{~m} 3$ de biogás diario con una concentración de $70 \%$ de metano, a partir de los residuales orgánicos generados del proceso productivo con un biodigestor anaerobio. La producción de biogás en las plantas que procesan cárnicos, resulta una alternativa adecuada, para propiciar una mejora de la eficiencia energética y las potenciales de la empresa, de manera sostenible y económica, con el fin de ahorrar y producir energías renovables,

Palabras clave: Eficiencia energética, Fuel Oíl, producción cárnica, digestor anaerobio, biogás.

\section{Abstract}

The aim of this research was to determine the consumption of electricity and Fuel Oil of a meat production company in Cuba. Existing information on the production and consumption of energy carriers was analyzed in a consecutive period of 36 months, applying the tools of the Total and Efficient Energy Management Techno-

Recibido: Junio 2020

Aceptado: Octubre 2020 logy (TGTEE), to establish the consumption of electric energy and Fuel Oíl and it was designed a fixed dome biodigester for biogas production. Electric energy and Fuel Oill were the major influences on the energy cost of production, representing $91.93 \%$ of total consumption in the evaluated period $15 \mathrm{~m} 3$ of biogas daily were obtained with a concentration of $70 \%$ methane, from the organic waste genera- 
ted from the production process with an anaerobic biodigester. The production of biogas in plants that process meat is a suitable alternative to promote an improvement in energy efficiency and the potential of the company, in a sustainable and economical way, in order to save and produce renewable energies,

Keywords: energy efficiency, Fuel Oíl, meat production, anaerobic biodigester, biogas.

\section{INTRODUCCIÓN}

Desde comienzos del presente siglo se ha reforzado el cuestionamiento del modelo energético vigente, que está basado preferentemente en los combustibles fósiles, como el petróleo, el carbón mineral y el gas natural. Resultan cada vez más evidentes los límites de dicho modelo, tanto en el orden económico (altos precios de la energía), como en el plano social (inequidad y pobreza energética) y ambiental (implicaciones adversas para el entorno) (Pichs., 2012).

A finales del siglo XIX en el mundo se consumían seis millones de toneladas de petróleo al año, mientras que, en la actualidad, el consumo diario es superior a los 12 millones de toneladas. En apenas un siglo, la población mundial ha crecido 6,5 veces y el consumo de energía se incrementó en más de 730 veces (Taliani y Álvarez, 1994, Hidalgo y Guerra, 2016).

En los últimos años se han reforzado los conflictos internacionales por el control de los recursos energéticos del planeta, sobre todo aquellos no renovables, como el petróleo y gas natural. Ante tales tendencias, se impone la necesidad de una reestructuración energética global que cambie significativamente los patrones actuales de generación y consumo de energía, y que promueva el desarrollo sostenible sobre las bases de equidad y justicia social. (Madruga., 2012, Pichs., 2012).

La energía está intrínsecamente vinculada a muchos de los objetivos de desarrollo sostenible, a la erradicación de la pobreza, la seguridad alimentaria, el agua potable y el saneamiento, el transporte y la movilidad sostenible (comunidades y ciudades sostenibles), la salud, la educación, la prosperidad, la creación de empleo etc. Por otra parte, el cambio hacia soluciones de energía sostenible, también es esencial para la consecución del Acuerdo de París aprobado por la Convención Marco de las Naciones Unidas sobre el Cambio Climático (CMNUCC), fundamentalmente relacionado con la emisión de gases y compuestos de efecto invernadero. (Becheran., 2013).

El panorama mundial actual precisa de consideraciones eficientes de consumo de energía, la competitividad productiva de los procesos industriales y de una visión económica ecologista, para poder garantizar la seguridad energética, productiva y medioambiental de las presentes y futuras generaciones (Taliani y Álvarez., 1994; Lander., 2011).

Dada la naturaleza global de los problemas económicos y ecológicos que se pretende resolver con una reestructuración energética sostenible, se requieren cambios radicales en los patrones de producción y consumo de energía a nivel global. No obstante, el ritmo y los patrones concretos de este proceso en cada región o país, dependerán de las condiciones específicas de cada caso.

Los patrones de una reestructuración energética sostenible se orientan en dos direcciones básicas:

- Incremento del ahorro y la eficiencia energética.

- Desarrollo de las fuentes renovables de energía.

La eficiencia energética consiste en la reducción de consumo de energía, manteniendo los mismos servicios energéticos, sin disminuir el confort ni la calidad de vida, asegurando el abastecimiento, protegiendo el medio 
ambiente y fomentando la sostenibilidad; no consiste únicamente en poseer las últimas tecnologías, sino de saber emplear y administrar los recursos energéticos disponibles de un modo hábil y eficaz, lo que requiere desarrollar procesos de gestión de la energía, en los que la introducción de fuentes de energías renovables juega un papel decisivo para el tránsito hacia la energética sostenible.

Los sistemas de Gestión Energética ofrecen un enfoque sistemático para controlar y reducir el consumo de energía de una organización. Desde hace años, los Sistemas de Gestión de la Energía se presentan como una herramienta para optimizar el uso y consumo de energía por parte de cualquier organización, no importa el sector de actividad y tamaño de que se trate.

Desde el año 2011, la ISO 50001 es la nueva norma global para los Sistemas de Gestión Energética, esta norma certifica la existencia de un sistema optimizado para el uso correcto de la energía en cualquier organización, sea cual sea su naturaleza o tamaño, su actividad o su dedicación, se basa en el ciclo de mejora continua Planificar - Hacer - Verificar - Actuar (PHVA) e incorpora la gestión de la energía a las prácticas habituales de la organización (Ver Figura 1)

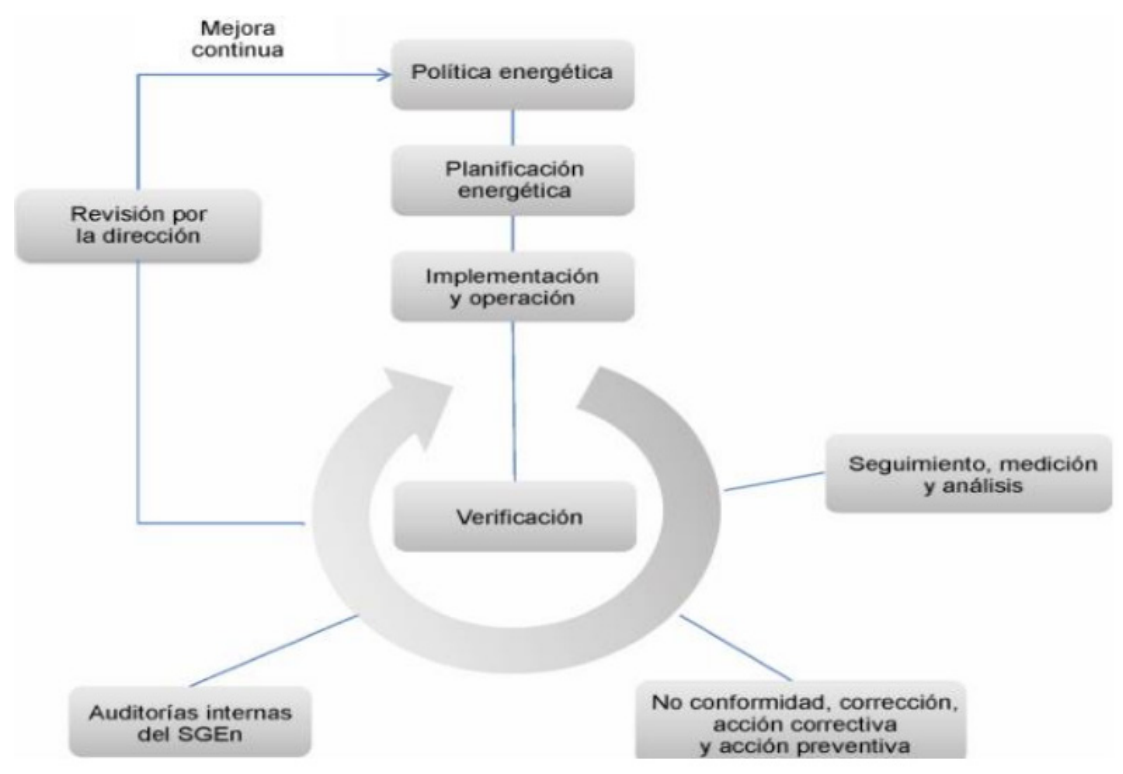

Figura 1. Modelo de sistema de gestión de la energía, (ISO 50001).

El Modelo de sistema de gestión de la energía para esta Norma Internacional está compuesto por:

- Política energética.

- Planeamiento energético

- Implantación y operación

- Comprobación

\section{Desarrollo de las fuentes renovables de} energía.

Varios estudios han demostrado la aplicabilidad de la digestión anaerobia para la producción de energía y el tratamiento de los residuales generados en las plantas procesadoras de cárnicos (Salminen y Rintala., 2002). La digestión anae- 
robia es una técnica atractiva, ya que tiene un alto potencial de tratamiento de los residuos orgánicos y ha tenido un creciente auge en los últimos años por ser una solución económicamente factible. Al mismo tiempo, tiene varios beneficios ambientales, los que incluyen: la producción de energía renovable en forma de biogás, la posibilidad de recirculación de nutrientes y la reducción de la contaminación (Aiyuk et al., 2006, Demirbas et al., 2011, Rao y Baral 2011., Zhang et al., 2013., Zhang et al., 2012).

La tecnología del biogás, es una forma de energía limpia y renovable y es un buen sustituto de las fuentes convencionales de energía (combustibles fósiles) (Yadvika et al., 2004). La composición típica del biogás es 55 - 70 \% de metano $\left(\mathrm{CH}_{4}\right), 30$ - $45 \%$ de dióxido de carbono $\left(\mathrm{CO}_{2}\right), 0$ - 2 \% de nitrógeno $(\mathrm{N})$ y trazas de otros compuestos, como por ejemplo el sulfuro de hidrógeno, denominado ácido sulfhídrico $\left(\mathrm{H}_{2} \mathrm{~S}\right)$. Entre estos compuestos, el metano es el más importante desde el punto de vista de combustión. Es una invaluable fuente de energía renovable, pero, también es un importante gas de efecto invernadero. El metano producido en el proceso de digestión anaerobia de los estiércoles presenta entre 4800 - $6700 \mathrm{kcal} \mathrm{m}^{-3}$, el cual es cercano a las $8900 \mathrm{kcal} \mathrm{m}^{-3}$ obtenida con la energía del metano puro (Rasi et al., 2007).

El biogás puede ser utilizado como combustible para los motores de combustión interna, las turbinas de gas, las celdas de combustible, los calentadores de agua, calentadores industriales, entre otros muchos procesos; así como, para la generación de electricidad, donde la eficiencia de conversión global está alrededor de 10 - $16 \%$ (Demirbas et al., 2011).

Este estudio tuvo como objetivo determinar el consumo de energía eléctrica y Fuel Oíl de una empresa de producción de cárnicos en Cuba

\section{MATERIALES Y MÉTODOS}

La UEB "Bayamo " de la Empresa Cárnica Granma, está ubicada en la carretera Central vía
Manzanillo, en la ciudad de Bayamo. Cuba. En la entidad se efectúa el sacrificio de ganado bovino y porcino, además de la elaboración de productos como carnes en conservas y masas cárnicas, para lo cual se utilizan varios portadores energéticos. Está situada en las coordenadas 20.3839, -76.65849.

Para evaluar la eficiencia de aprovechamiento de los recursos energéticos asociados a los diferentes procesos productivos; así como, la caracterización energética de la empresa, se aplicó la Tecnología de Gestión Total Eficiente de Energía (TGTEE), (CEEMA.,2002; Borroto., 2009). El periodo evaluado fue de 36 meses de trabajo continuo.

\section{Herramientas para establecer un sistema de gestión total eficiente de la energía.}

El objetivo de utilizar estas herramientas es que, permiten implementar las secuencias propuestas por la tecnología, por su nivel de complejidad generalmente se clasifican en básicas, medias y avanzadas.

\section{Herramientas Básicas.}

Las herramientas básicas son aquellas que se sustentan en métodos estadísticos que permiten desarrollar un proceso deductivo, el cual va de lo general a lo particular, detectando las causas de los problemas a partir de datos de consumo y producción suministrados por la empresa, se puedan generar, por una parte, las condiciones de operación típicas para la empresa, y por otra, indicadores de comparación. También proporcionan el análisis de un mismo problema con diferentes criterios lográndose una visión más completa del mismo (Restrepo., 2003). Estas deben ser de conocimiento de todos los miembros de un círculo o grupo de calidad (Borroto.,2009).

El empleo de estas herramientas básicas, sigue el principio de Pareto, por consiguiente, unas pocas de ellas, permiten resolver la mayor parte de los problemas. Las herramientas básicas utilizadas para la investigación fueron las siguientes: 
- Diagrama de Pareto.

- Estratificación.

- Histogramas.

- Gráficos de control.

- Diagrama causa y efecto.

- Diagrama de diszersión.

Después de aplicadas las herramientas de nivel 1 , se buscaron las potencialidades de ahorro y/o producción de nuevas fuentes energéticas a partir del potencial existente en la propia entidad, determinando que en la misma existe un potencial considerado de producción de biogás a partir de los residuales de los animales sacrificados, que puede ser utilizado fundamentalmente en la generación de vapor.

\section{Potencial de producción de biogás.}

\section{Residuos generados.}

Los residuales generados en la entidad contienen: sangre, orina, pequeñas partículas de carne, restos de tejidos y alimentos contenidos en el interior del estómago de los animales sacrificados; así como, restos de las materias primas utilizadas en la elaboración de los diferentes conformados (condimentos, grasas vegetales, harina, entre otros). Las áreas de mayor influencia en la generación de residuales son las de sacrificio y preparación, debido a la gran cantidad de agua que se emplea en los procesos productivos que desarrollan: lavado de los animales, productos y subproductos, instrumental de proceso, envases entre otros. Otra área importante de generación de residuos son los corrales, donde con agua se recolecta el estiércol producido por los animales.

\section{Metodología de diseño de los biodiges- tores de cúpula fija.}

Para el cálculo de los parámetros de diseño del biodigestor anaerobio se utilizó la metodología expuesta por Thi-Thanh et al., 2005.

\section{RESULTADOS Y DISCUSIÓN}

Diagrama de Pareto.

El principio de Pareto, demuestra que, en el caso de estudio, la energía eléctrica con un $58,04 \%$ y el Fuel Oíl, con un 33,90 \% son los que más influyen dentro de los consumos energéticos, representando en el periodo evaluado, entre ellos, un acumulado de 91,93 $\%$ del total de los portadores energéticos utilizados en el proceso productivo (Figura 2). Ello demuestra que el programa de ahorro de energía debe tener su mayor accionar en estos portadores, para lograr mejores resultados en menores intervalos de tiempo.

Estructura de Consumo de portadores Energéticos

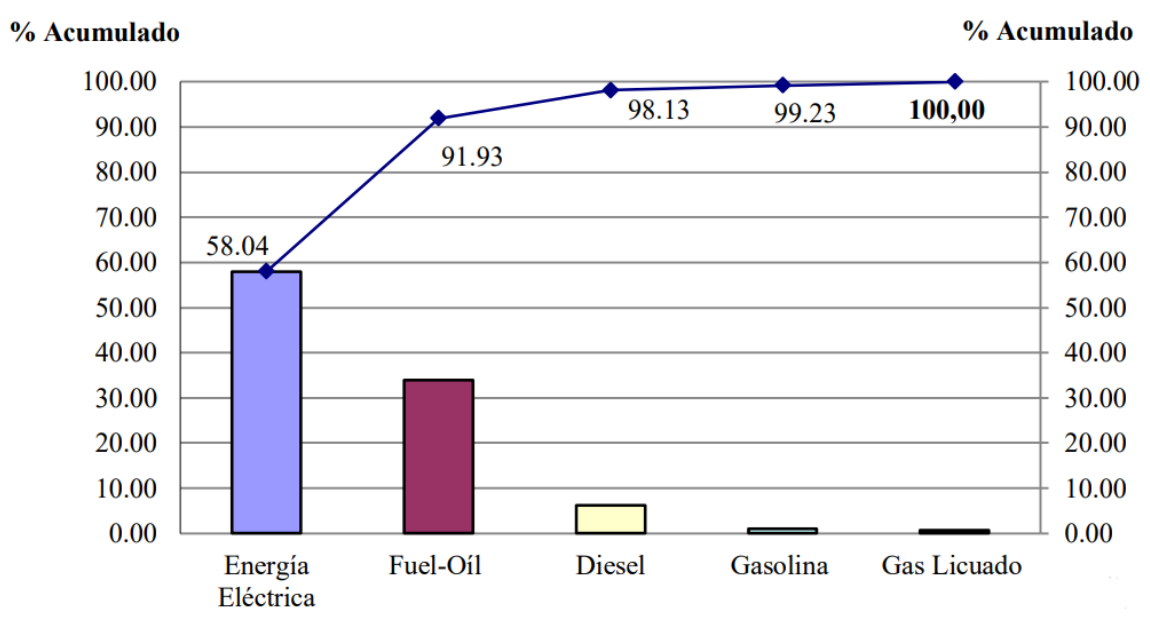

Figura 2. Diagrama de Pareto de portadores energéticos. 
El estudio detallado por áreas de la empresa permitió determinar los puestos claves asociados al consumo de estos dos portadores energéticos. La energía eléctrica tiene los mayores consumos en el proceso de refrigeración y en la producción, siendo esta última la de mayor influencia; y asociado al consumo de Fuel Oíl, el cual se utiliza solo en la generación de vapor, el área de producción. Se determinan como puestos claves los procesos de refrigeración y producción de la empresa, en los cuales se deben concentrar los esfuerzos para disminuir los consumos y hacer más eficiente energéticamente la empresa.

Gráficos de Control

Después de determinar los portadores que

\section{$\left(M W{ }^{*}\right.$ h Control del consumo de Energía Eléctrica}

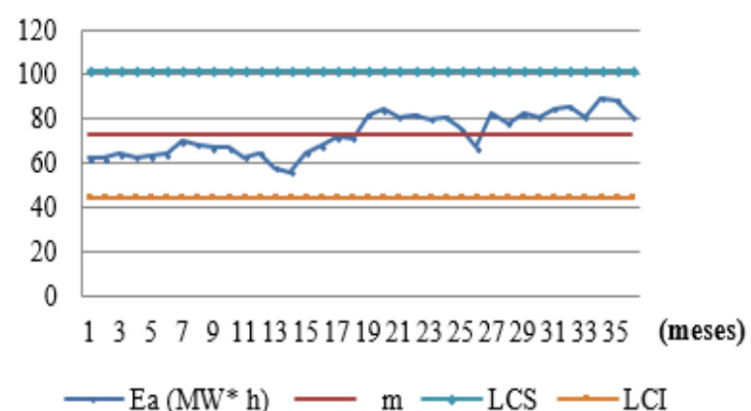

Figura 3. Control del consumo de energía eléctrica.

En las figuras 5 a la 10 se muestran los comportamientos del consumo de energía eléctrica; así como, su correlación en los 36 meses evaluados, en periodos de 12 meses. En ellos, se aprecia que no existe una relación fuerte entre el consumo de energéticos y la producción, ya que en todos los casos existen irregularidades que se visualizan a partir del crecimiento de uno de los indicadores con una consecuente disminución del otro, negándose en estos casos las funciones teóricas que describen este tipo de procesos y sus valores de $\mathrm{R}^{2}$, que arrojan que la relación en- representan al menos el $80 \%$ de los gastos energéticos asociados al proceso productivo, se analiza el comportamiento de cada uno de los involucrados en este de manera independiente, para determinar de qué manera mejorar su gestión eficiente.

Al realizar un análisis de los gráficos de control (Figuras 3 y 4 ), se pudo verificar que los consumos de energía eléctrica y Fuel Oíl, se mantienen dentro de los límites de control superior (LCS) e inferior (LCI) y que, aunque no se sobrepasan estos límites, existen anomalías en el uso del portador que implican buscar las causas que las propician.

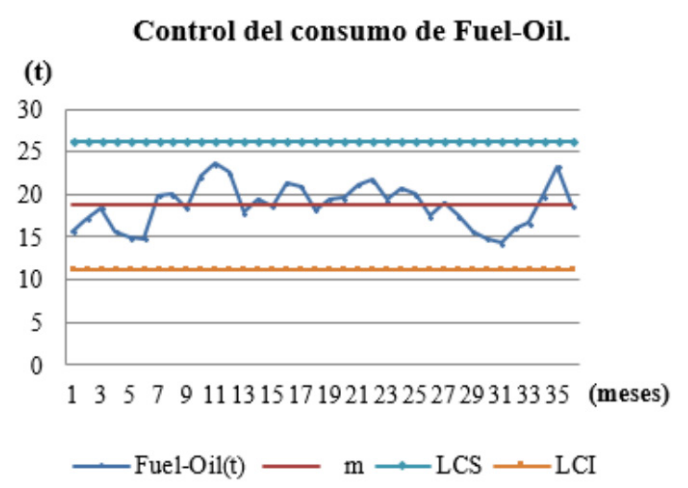

Figura 4. Control del consumo de Fuel Oíl.

tre los factores evaluados es débil y la gestión del recurso energético no es buena, pues $R^{2}$ es menor que el $75 \%$; es decir, no existe una relación bien definida entre el consumo y la producción, obteniendo valores del $2 \%$, el 70 $\%$ y el $19 \%$ respectivamente, lo que connota la necesidad de tomar medidas de ahorro y eficiencia energética en aras de mejorar lo mismos.

En las Figuras 5, 7 y 9 se pueden visualizar que los gastos de energía eléctrica no asociados a la producción son elevados; así como, existen 
disminuciones en la producción de la empresa en los cuales existe una tendencia a mantener los consumos elevados, lo cual pudiera estar asociado a lluminación de plantas, electricidad para equipos de oficinas, ventilación,

\section{Consumo Electricidad y Producción.}

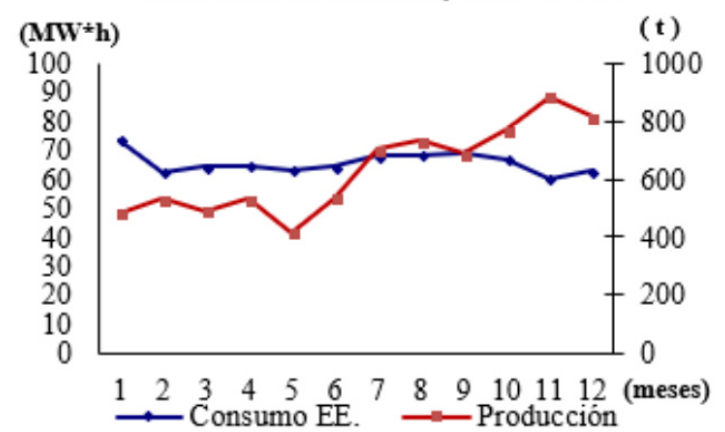

FIGURA 5. Consumo de energía eléctrica y producción.

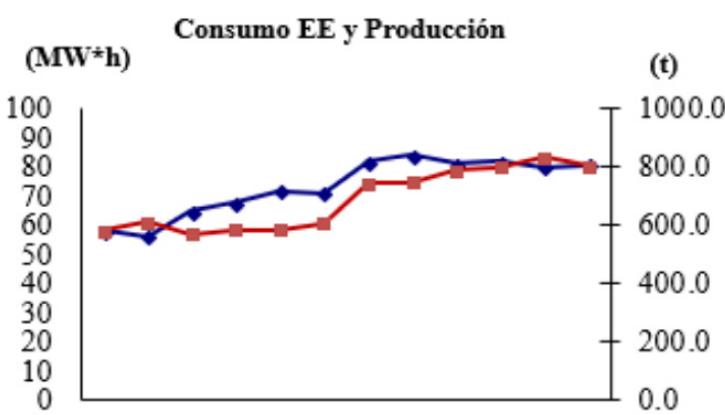

131415161718192021222324 (meses) $\longrightarrow$ Consumo EE.

- - Producción

FIGURA 7. Consumo de energía eléctrica y producción.

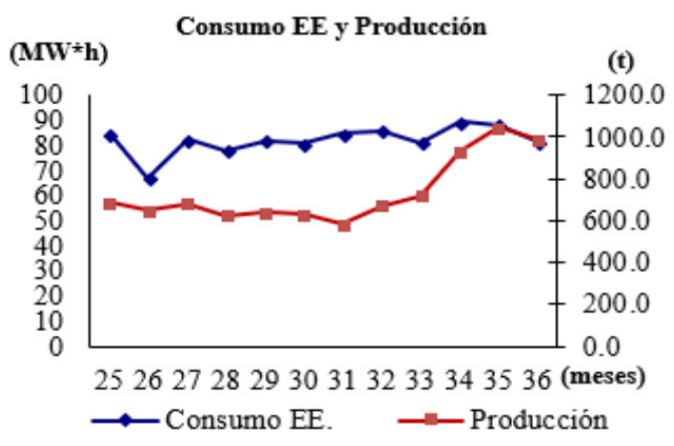

FIGURA 9. Energía eléctrica y producción. áreas climatizadas, energía usada en servicios de mantenimiento, trabajo en vacío de equipos eléctricos o térmicos, pérdidas de electricidad por potencia reactiva (Fernández.,2006; Borroto.,2009; Lescay.,2010; Linares.,2012).

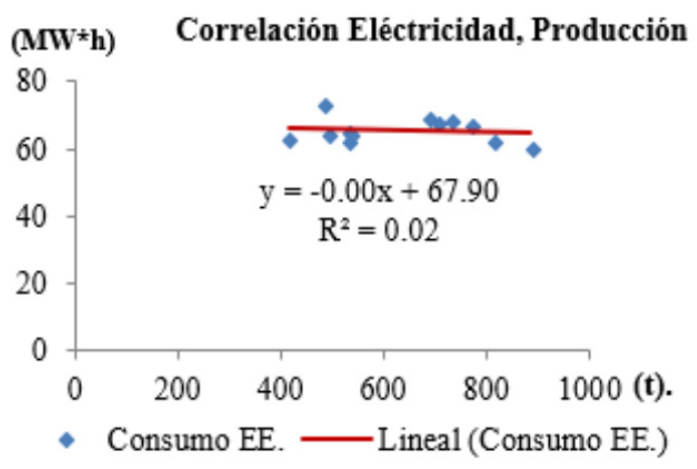

FIGURA 6. Correlación entre consumo de energía eléctrica y producción

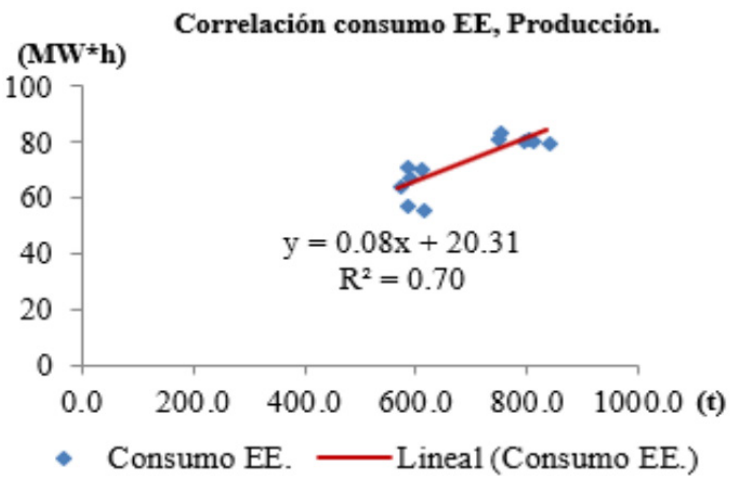

FIGURA 8. Correlación entre consumo de energía eléctrica y producción

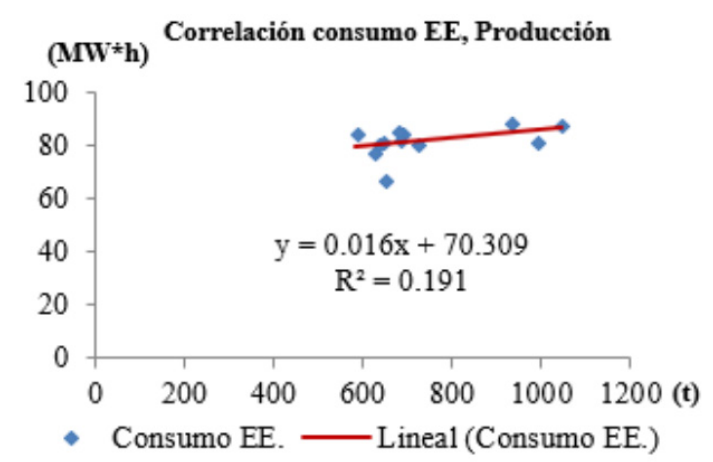

FIGURA 10. Correlación entre consumo de energía eléctrica y producción 
Las figuras 11 a 16 muestran la variación simultánea del consumo de Fuel Oíl con la producción realizada en el tiempo, se observa que existe una buena correspondencia entre el consumo activo de Fuel Oíl y la producción, exceptuando los meses 1, 6,11, 13, 19, 23, 25, 31 y 35. También se observan elevados consu-

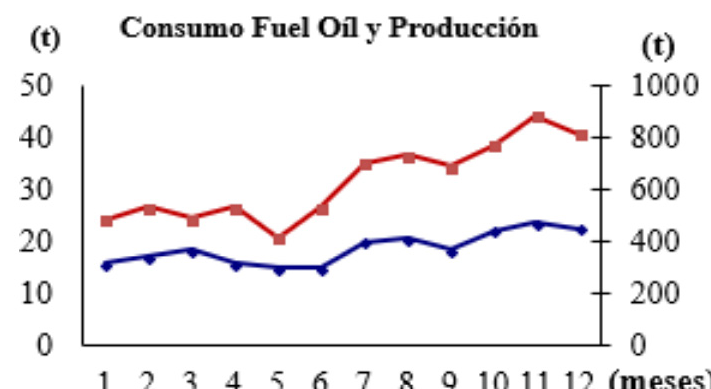

$\begin{array}{llllllllllll}1 & 2 & 3 & 4 & 5 & 6 & 7 & 8 & 9 & 10 & 11 & 12 \text { (meses) }\end{array}$

Consumo Fuel Oíl $\quad \longrightarrow$ Producción

FIGURA 11. Variación del consumo de Fuel Oíl y producción.

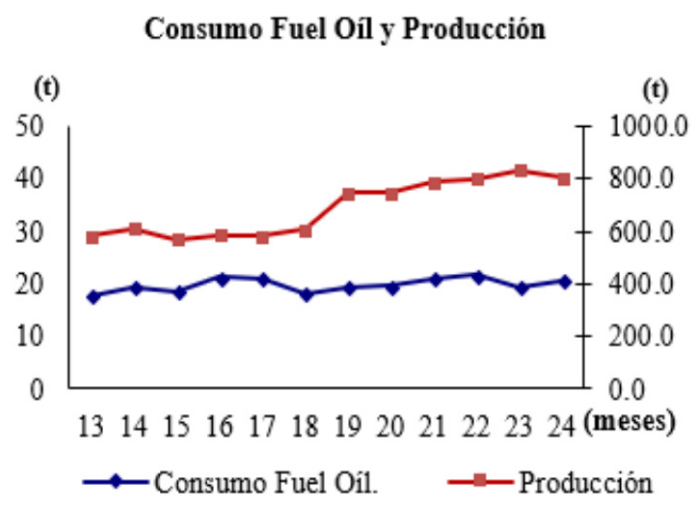

FIGURA 13. Consumo de Fuel Oíl y producción.

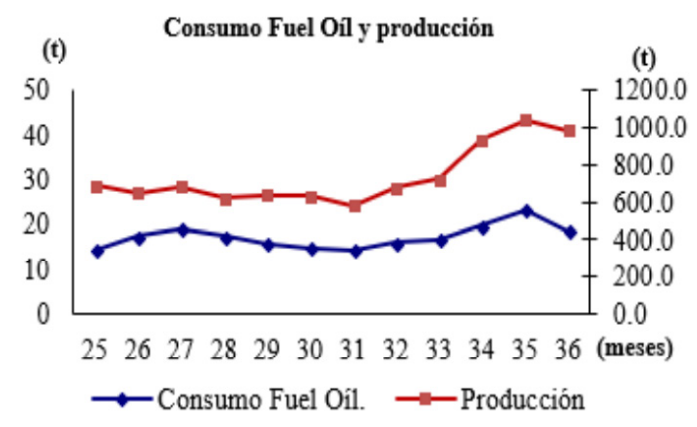

FIGURA 15. Variación del consumo de Fuel Oíl y producción. mos no asociados al proceso de producción y fugas de energía asociadas a energía perdida en salideros de vapor, deficiente aislamiento térmico, pérdidas por radiación y convección en calderas y precalentamiento de equipos y sistemas de tuberías (Restrepo.,1999; BorreII.,2007).

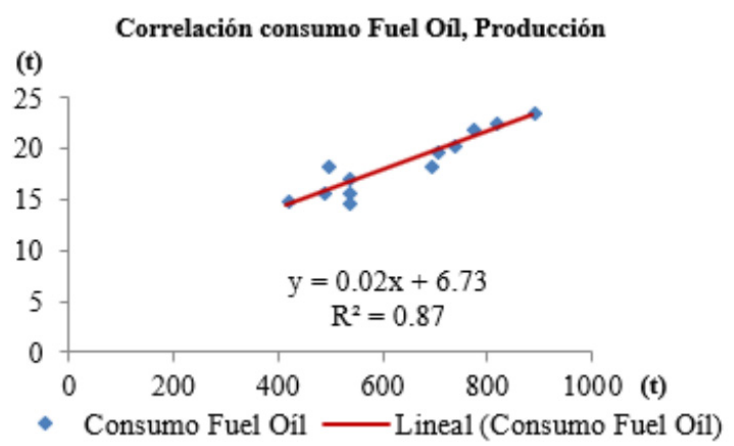

FIGURA12. Correlación entre consumo de Fuel Oíl y producción.

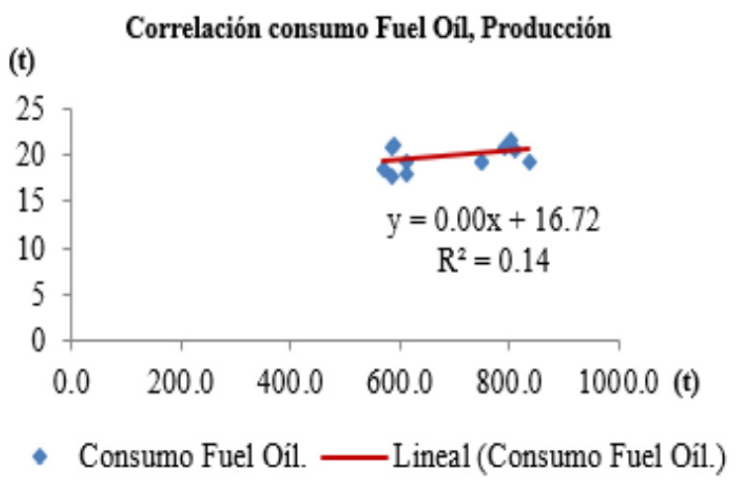

FIGURA14. Correlación entre consumo de Fuel Oíl y producción.

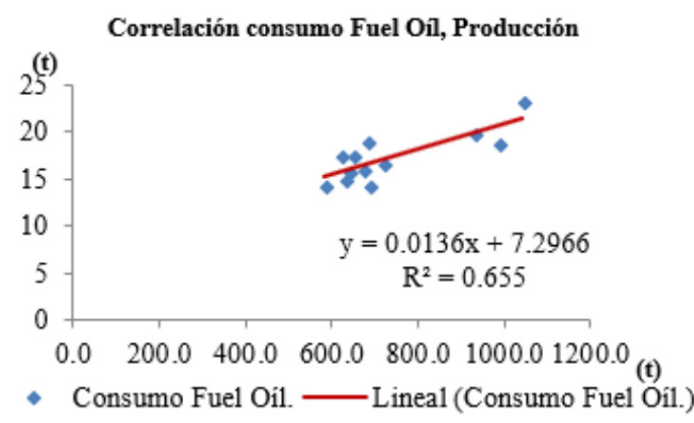

FIGURA16. Correlación entre consumo de Fuel Oíl y producción 
La correlación entre el consumo de Fuel Oíl y la producción (Figuras 12, 14 y 16), arroja que, aunque en los primeros 12 meses la gestión del recurso fue buena, en los años siguientes esta se perdió, debido a que los valores de $\mathrm{R}^{2}$ caen a un $14 \%$ y aunque en el periodo de 25 a 36 meses se recuperó hasta el 65 \%, también la gestión de este recurso no fue buena durante este periodo de tiempo.

\section{Diseño del biodigestor anaerobio para la producción de biogás.}

Considerando que se realiza una dilución de la materia orgánica de 1:3 (una parte de residuo sólido y tres partes de agua) se tiene una cantidad de materia diaria aproximada de $3 \mathrm{~m}^{3} \mathrm{~d}^{-1}$, con un tiempo de retención de 30 d, el volumen total del biodigestor calculado es de $90 \mathrm{~m}^{3}$. Con este volumen se procedió a la selección del biodigestor con volúmenes prototipos, los cuáles comprenden volúmenes de $10 \mathrm{~m}^{3}, 14 \mathrm{~m}^{3}, 22,5$ $m^{3}, 42,5 m^{3}$ y $47,5 m^{3}$ (Guardado., 2007).

Con los volúmenes de los biodigestores obtenidos mediante el cálculo, y utilizando los diseños prototipos, se procedió a la selección de los volúmenes reales y sus parámetros constructivos, por lo que se decidió la selección de dos plantas de 47,5 m³, para garantizar un mejor funcionamiento de las mismas; además, de poder dar mantenimientos, sin que se afecte el proceso de producción de biogás y de tratamiento de los residuales.

\section{Producción de Biogás.}

La producción de biogás se determinó aplicando la ecuación que a continuación se relaciona, obtenida a partir del estudio de biodegradabilidad anaerobia de los residuales de la entidad (Guardia et al., 2017).

Producción de metano $=1501$ Estiércol $+948,1$ Rumen + 560,3 Agua residual - 1962 Estiércol*Rumen - 1,37.104 Estiércol*Rumen*Agua residual (2.10)

Después de aplicar la ecuación se obtuvo que la producción predicha diaria de biogás a obtener, a partir del tratamiento de los residuales sólidos de la UEB Cárnicos Bayamo en reactores del tipo chino GBV, fue de aproximadamente $15 \mathrm{~m}^{3}$ diarios, con un $70 \%$ de concentración de metano.

\section{CONCLUSIONES}

- Los portadores energéticos que más inciden en los consumos de la UEB Cárnicos son la energía eléctrica y el Fuel Oíl representando un $91.93 \%$ del total de los gastos en el período evaluado.

- La situación actual de la UEB muestra que los niveles de eficiencia energética son bajos.

- La correlación lineal entre los consumos y la producción es débil.

- Existe un potencial para la producción de $15 \mathrm{~m}^{3}$ diarios de biogás para la generación de vapor si se construyen los dos digestores de $47,0 \mathrm{~m}^{3}$ de digestión.

\section{REFERENCIAS BIBLIOGRÁFICAS}

Aiyuk, S; Forrez, I; deLieven, K; vanHaandle, A; Verstraete, W. (2006). Anaerobic and complementary treatment of domestic sewage in regions with hot climates - A review. Bioresource. Technology, 97(17), 2225-2241. ISSN: 09608524, doi: 10.1016/j.biortech.2005.05.015.

Becheran, JM, (2013). Potencialidades para el ahorro de energía eléctrica en el área de basculadores y molinos de la UEB azucarera Antonio Guiteras Holmes. Santiago de Cuba.

Borrell, E. (2007). Sistema de Gestión Total Eficiente de la Energía en la CTE Carlos M. de Céspedes. Cienfuegos.

Borroto, AE. (2009). Tecnología de Gestión Total Eficiente de la Energía. Cienfuegos, Cuba: Editorial Universo Sur.

Bravo HD. (2015). Energía y desarrollo sostenible en Cuba. Centro Azúcar, 42(4), 14-25.

Demirbas, MF; Balat, M; Balat, H. (2011). Biowastes-to-biofuels. Energy Conversion and Management, 52, 1815-1828. ISSN: 
0196-8904,doi: $\quad$ http://10.1016/j.enconman.2010.10.041.

Fernández, ADF. (2006). Eficiencia energética. Modelo cubano de empleo sostenible de la energía eléctrica.

Guardado, ChJA. (2007). Diseño y construcción de plantas de biogás sencillas. Ciudad de la Habana, Cuba: Editorial Cubasolar.

Guardia, PY; Herrera, RM; Rodríguez, PY; Rodríguez, PS; Pérez CM. (2017). Statistical modeling of the methane production from slaughterhouse wastes in anaerobic co-digestion. British Journal of Environment \& Climate Change, 7(1), 13-25. ISSN: 2231-4784,doi: http://dx.doi.org/10.9734/BJECC/2017/29741

Lander, E. (2011). La Economía Verde: el lobo se viste con piel de cordero. Transnational Institute. Retrieved from https://www.tni.org/en/ publication/the-green-economy-the-wolf-insheeps-clothing?content_language=es

Lescay, NR. (2010). Gestión de Eficiencia Energética en la Unidad Empresarial Económica Ferroviaria de base en Ciro Redondo, Ciego de Ávila.

Linares, SC. (2012). Energía e indicadores de desarrollo energético sostenible. CUBAENERGÍA.

Madruga., RP. (2011). Tendencias energéticas mundiales: implicaciones sociales y ambientales. Disponible: http://www.cubasolar.cu/biblioteca/Ecosolar/Ecosolar20/HTML/articulo01.htm

Madruga., RP. (2012). Principales tendencias y retos energéticos globales en 2011-2012.

Mestre., LRM. (2010). Gestión Energética en el Policlínico Juan Olimpio Valcárcel del municipio Venezuela. Ciego de Ávila.

Norma internacional ISO 50001. 2013.

OIEA. (2008). Indicadores energéticos del desarrollo sostenible: directrices y metodologías.
Pérez, LB. (2014). Soberanía energética, medio ambiente y desarrollo local sostenible.

Rao, PV; Baral, SS. (2011). Experimental design of mixture for the anaerobic codigestion of sewage sludge. Chemical Engineering Journal, 172, 977-986.doi: http://10.1016/j. cej.2011.07.010.

Rasi, S; Veijanen, A; Rintala, J. (2007). Trace compounds of biogas from different biogas production plants. Energy, 32, 1375-1380. https://doi.org/10.1016/j.energy.2006.10.018

Restrepo, VÁ H. (1999). Memorias de diplomado Gestión Total Eficiente de la Energía, Universidad de Cienfuegos. Cienfuegos, Cuba.

Salminen, E; Rintala, J. (2002). Anaerobic digestion of organic solid poultry slaughterhouse waste - a review. Bioresource Technology, 83, 13-26.

Thi TH; Pham TK, et al. (2005). Tecnología del Biogás. Manual para la Formación. Ha Noi. Viet Nam.

Yadvika, S; Sreekrishnan, TR; Kohli, S; Rana, V. 2004. Enhancement of biogas production from solid substrates using differents techniques - a review. Bioresource Technology, 95, 1-10. doi: 10.1016/j.biortech.2004.02.010.

Zhang, T; Liu, L; Song, Z; Ren, G; Feng, Y; Han, X; Yang, G. 2013. Biogas Production by Co-Digestion of Goat Manure with Three Crop Residues. PLOS ONE, 8(6), 1-7. ISSN: e66845, doi: 10.1371/journal.pone.0066845.

Zhang, Y; Banks, C; Heaven, S. 2012. Anaerobic digestion of two biodegradable municipal waste streams. Journal of Environmental Management, 104, 166-174. ISSN: 0301-4797, doi: 10.1016/j.jenvman.2012.03.043.

\section{CORRESPONDENCIA:}

Dr.C. Yoandro Rodríguez Ponce

ypoonce@udg.co.cu 